\title{
Reaction tubes as a platform for silicon nanophotonic ring resonator biosensors
}

\author{
C. Lerma Arce ${ }^{\text {a-c }}$, A. Goes ${ }^{\text {b-c }}$, E. Hallynck ${ }^{\text {a-c }}$, P. Dubruel ${ }^{\text {b-c }}$, K. Komorowska ${ }^{\text {a-c }}$, S. Van Put ${ }^{\text {d }}$, P. Bienstman ${ }^{\text {a-c }}$ \\ ${ }^{a}$ Photonics Research Group (INTEC) Gent University, Sint-Pietersnieuwstraat 41, 9000 Gent, Belgium \\ ${ }^{b}$ Polymer Chemistry and Biomaterials Group, Gent University, Krijgslaan 281 (S4), 9000 Gent, Belgium \\ ${ }^{c}$ Center for Nano- and Biophotonics, Gent University, 9000 Gent, Belgium, \\ ${ }^{d}$ Center for MicroSystems Technology, Gent University, Technologiepark 914, 9052 Zwijnaarde (Gent), Belgium \\ Cristina.Lermaarce@intec.ugent.be
}

\begin{abstract}
We propose the combination of a simple reaction tube platform with label free SOI photonic biosensors. The device allows for the excellent performance of ring resonator sensors in a user-friendly platform to be used in labs and hospitals.

OCIS codes: (280.0280) Remote sensing and sensors (2801415) Biological sensing.
\end{abstract}

\section{Introduction}

Label-free biosensing with silicon nanophotonic microring resonator sensors has proven to be an excellent sensing technique for achieving high-throughput and high sensitivity, comparing favorably with other labeled and label-free sensing techniques. However, as in any biosensing platform, silicon nanophotonic microring resonator sensors require a fluidic component which allows the continuous delivery of the sample to the sensor surface. This is the big disadvantage of this platform since this type of microfluidic system is very much removed from the daily practice in e.g. hospital labs, which still relies to a large degree on platforms like 96-well microtiter plates, or reaction tubes.

Here we propose the combination of a simple and lab-compatible reaction tube platform, with label-free nanophotonic biosensors with a special microfluidic system imbedded in the same chip, where the flow is through the chip as opposed to over the chip as in more traditional approaches. This shows that label-free nanophotonic ring resonators can be also used in the user-friendly platform like reaction tubes or well microtiter plates, conserving their excellent performance

\section{Nanophotonic ring resonator sensors, chip layout, and setup}

The photonic chip was fabricated in SOI with $2-\mu \mathrm{m}$ buried oxide and a $220-\mathrm{nm}$ silicon top layer with CMOS compatible 193-nm optical lithography and dry etching [1]. The ring resonators consist of 450-nm-wide single-mode waveguides, with $5-\mu \mathrm{m}$ bend radius, $2-\mu \mathrm{m}$-long directional couplers, and a gap of $180 \mathrm{~nm}$ between the waveguides. The layout of the chip is illustrated in Figure. 1.

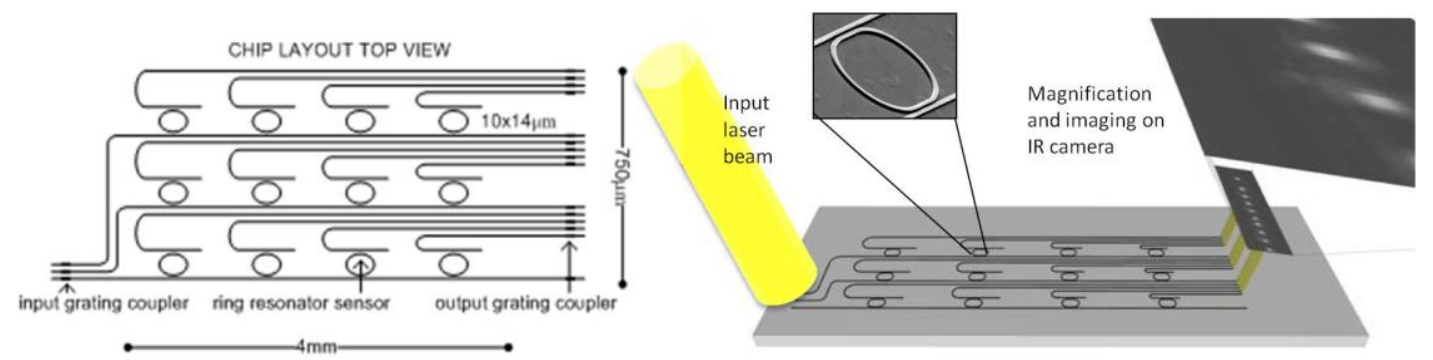

Figure 1. Left. Chip layout top view. Four rings are connected to one common input waveguide, each of them having a dedicated drop signal port. Three of these four ring series are placed independently next to the other. Right: Setup: A SANTEC TSL-510 tunable laser was used as a light source. The output signals of the ring resonators are imaged with an infrared camera [2].

Four rings are connected to one common input waveguide, each of them having a dedicated drop signal port. Three of these four ring series are placed independently next to the other. The three input waveguides are simultaneously addressed through vertical grating couplers [3] with a 2-mm-wide collimated beam from a tunable laser source. The output signals of the ring resonators are near-vertically coupled to free space by means of integrated grating couplers and are imaged with an infrared camera.

A TSL-510 tunable laser is used as a light source. The transmitted light is detected by an infrared camera. We have developed software that captures an image for every wavelength step and stores the maximum intensity values within each dedicated area that overlaps with an output grating coupler spot. Postprocessing consists of fitting the 
spectra to their theoretical shape and tracking these resonance peaks over time. This optical setup allows very high alignment tolerances, measures the spectrum of all the ring resonators in parallel, and therefore presents no limitation for high-throughput sensing.

\section{Embedded microfluidic system}

We propose a microfluidic system embedded in the photonic chip that will be integrated at the bottom of the reaction tube. This microfluidic system consists of certain apertures that perforate the chip from the top to the bottom. The solution inserted in the tube will flow through these openings that work as exit channels, creating a flow, which will accelerate the detection process. Figure 2 shows a schematic of the device with the embedded microfluidic system.

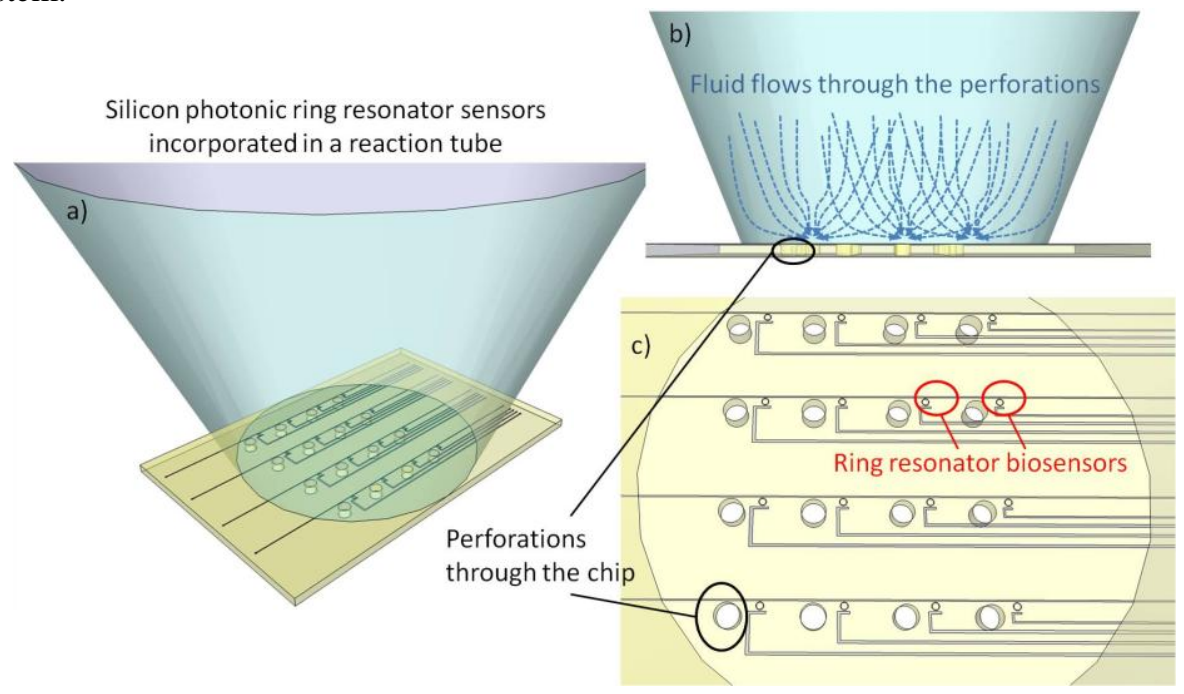

Figure 2. a) Schematic of the device. The silicon-on-insulator chip with the photonic biosensors and the embedded microfluidic system is incorporated at the bottom of a reaction tube. b) The solution inserted in the tube will flow through these openings that work as exit channels, creating a flow, which will accelerate the detection process. c) Layout of the chip. The array of ring resonators sensors is accompanied by an array of perforations next to them.

A Duetto laser source (Time-Bandwidth) was used to perform the perforations of the silicon-on-insulator chip by means of laser ablation. $1000 \mathrm{ps}$-duration pulses were applied with a repetition rate of $50 \mathrm{kHz}$ at $355 \mathrm{~nm}$. The size of the openings and their position can be easily optimized by editing some parameters in the laser.

\section{Experiments and results}

The photonic chip with the array of sensors and the embedded microfluidic system described above is incorporated to the bottom of the reaction tube, once its original bottom is mechanically removed. The attachment of the chip to the bottom of the tube is done permanently using UV curable glue which allows us to align precisely the array of sensors in the center of the reaction tube.

To perform the experiments, the device was fixed on a tiny chuck by means of vacuum. This chuck also has a connection to a pump, where pressure can be applied positively or negatively, pushing or sucking any gas of fluid applied in a specific area of this chuck. When the fluid under analysis will be inserted in the tube, this will be in contact with the sensors, and flow out through the holes.and be sucked or pushed up again by the pump. Figure 6a shows the device fixed on this chuck.

A new aspect with respect to our previous work [2] is that now, we have the possibility of coupling light in and out from the bottom of the chip, i.e. through the silicon substrate. (Figure 6b) Silicon is considered practically transparent for the wavelength used $(1.55 \mu \mathrm{m})$, however a few simple processing steps need to be done in advance to facilitate the alignment and detection of light. 


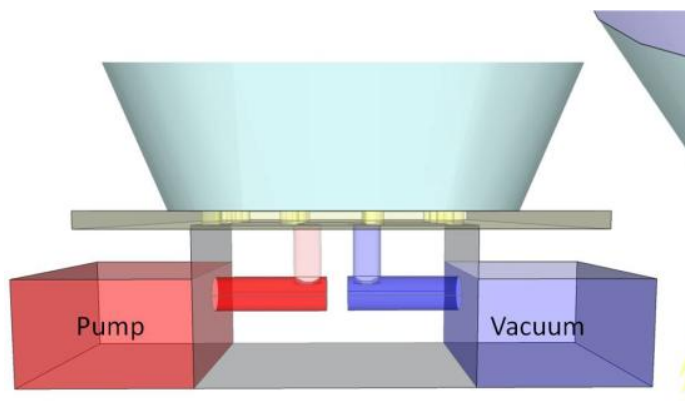

a) Chuck with a connection to vacuum and to a pump where pressure can be applied.

b) Light is coupled in and out through

the chip substrate.

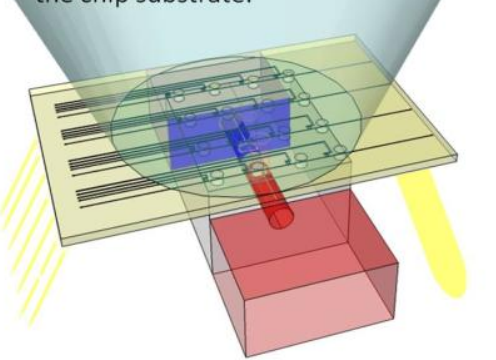

Figure 6. a) Device mounted on the chuck with a connection to vacuum and to a pump, where pressure can be applied in order to suck or push the fluid on the chip surface. b) The device is fixed to the chuck with vacuum. The measurements are performed from the bottom of the chip, i.e. light is coupled in and out through the chip substrate.

As a proof-of-principle to show the capabilities of the combined device, we measured the different steps for an assay where the well-known high-affinity couple biotin-streptavidin was bound. The measurements were performed as follows: Small volumes of different solutions were manually pipetted in the tube, and they were sucked out through the perforations of the bottom of the tube while being measured. The experiment consists of three different steps: silanization of the surface with aminosilane 3-Aminopropyl)triethoxysilane (APTES), immobilization of biotin and binding of streptavidin. Figure 8 shows three different graphs corresponding to each one of the steps of the assay. They show the evolution in time of the resonance wavelength shift of the ring resonators during the measurements with different solutions. Each color corresponds to one sensor.
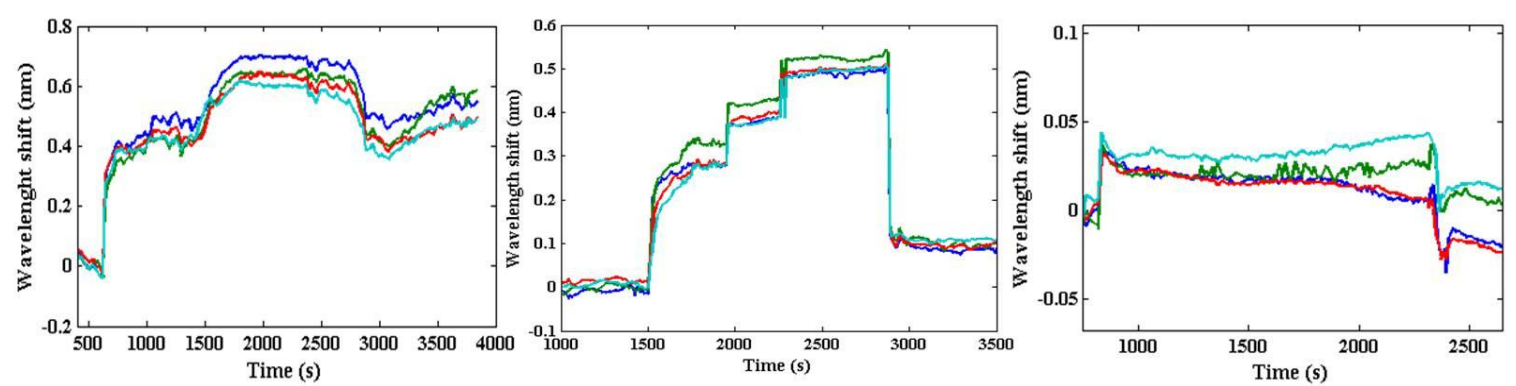

Figure 8. Three different steps of the bioassay were measured. From left to right. a) Silanization of the surface with APTES: ethanol-APTES- ethanol b) inmobilization of the biotin: PBS- biotin- PBS. c) binding of streptavidin to biotin: PBS- streptavidin- PBS

The association and disassociation of APTES in the sensor is easily quantifiable in Figure 8 Left. Figure 8 Center shows the binding of biotin after flowing $3 \mathrm{mg} / \mathrm{ml}$ solution of biotin in PBS and its disassociation when it is rinsed with PBS. A shift of $30 \mathrm{pm}$ is measured when streptavidin $(0.1 \mathrm{mg} / \mathrm{ml}$ in PBS) is flowed through the chip proving the binding of this to the biotin.

These measurements are preliminary results for the device just developed. Future work will imply the optimization of the device and the measurements.

\section{Conclusions}

We have presented the combination of a simple and compatible reaction tube platform, with label free nanophotonic biosensors and a special microfluidic system embedded in the same chip, showing that label-free nanophotonic ring resonators can also be used in a user-friendly platform like reaction tubes or well microtiter plates, conserving their excellent performance.

[1] S.K. Selvaraja, P. Jaenen, W. Bogaerts, D. Van Thourhout, P. Dumon, and R. Baets, “,” J. Lightwave Technol 27: 4076-4083 (2009)

[2] K. De Vos, J. Girones Molera, T. Claes, Y. De Koninck, S. Popelka, E. Schacht, R. Baets, P. Bienstman,” Multiplexed antibody detection with an array of silicon-on-insulator microring resonators," IEEE Phot Journal, 1(4): 225-235 (2009)

[3] D. Taillaert, P. Bienstman, R. Baets, "Compact efficient broadband grating coupler for silicon-on-insulator waveguides”, Optics Letters, 29(23):.2749-2751 (2004) 